\title{
The diagnosis of pulmonary embolism
}

\author{
A. Celi, L. Marconi, L. Villari, A. Palla
}

ABSTRACT: The diagnosis of pulmonary embolism. A. Celi, L. Marconi, L. Villari, A. Palla.

The diagnosis of pulmonary embolism is challenging, and autoptic series have demonstrated that a high percentage of cases are not recognized ante-mortem. A number of predisposing factors, symptoms and signs associated with pulmonary embolism have been recognized, and should be used to raise the suspicion of the disease. These include immobilization, recent surgery, active cancer, previous thromboembolism, syncope, dyspnoea, chest pain, haemoptysis, signs of deep vein thrombosis, hypocarbic hypoxemia. Once pulmonary embolism is suspected, the clinical probability of the disease should be assessed; to this end, three clinical rules have been proposed and validated (the revised Geneva score, the Wells score and the PISA-PED score) while others await clinical validation. In case of low clinical prob- ability, a negative a D-dimer test is sufficient to rule out the diagnosis, while if the clinical probability is high, or the Ddimer test is positive, further tests are necessary. Computer tomography angiography or perfusion lung scan are the imaging tests of choice, depending on local availability and experience. If the clinical probability and the results of the imaging test are concordant, a definitive diagnosis can be obtained; if the results are discordant, further testing is necessary. In particular, in the specific case of a small clot (i.e. segmental or subsegmental) incidentally recognized at a computer tomography obtained for other reasons in a patient without a clinical suspicion of pulmonary embolism, an occurrence whose frequency is rapidly increasing in clinical practice, a final diagnosis cannot be made without further confirmatory testing.

Monaldi Arch Chest Dis 2009; 71: 2, 47-53.

Keywords: Pulmonary embolism, Clinical probability, Lung scan, Computed tomography angiography.

Dipartimento Cardiotoracico e Vascolare, University of Pisa, Italy.

Correspondence: Prof. Antonio Palla, Dipartimento Cardiotoracico e Vascolare Azienda Ospedaliero-Universitaria Pisana Via Paradisa, 56124 Pisa, Italy; e-mail: a.palla@med.unipi.it

Pulmonary embolism (PE) is a common and potentially life threatening disease. Studies carried out in the US and in Europe have estimated the incidence of $\mathrm{PE}$ at approximately 60-80 cases/100,000 per year $[1,2]$. We had previously estimated the incidence of PE in the Pisa (Italy) area at 100 new cases/100,000 per year [3].

Anticoagulation with heparin or heparin derivatives, usually followed by oral vitamin $\mathrm{K}$ antagonists, represents an effective and relatively safe therapeutic approach [4]. Indeed, the landmark report by Barritt and Jordan had shown a clear benefit in terms of survival with early anticoagulation almost 50 years ago [5]. Nevertheless, bleeding does occur in patients undergoing anticoagulant therapy, and is potentially severe [6].

The importance of a prompt and accurate diagnosis of PE emerges clearly from the above considerations, since underdiagnosis deprives the patients of a very effective, potentially life saving therapy, while overdiagnosis exposes them to unjustified risk of bleeding. Unfortunately, such goal is still largely unmet. Data from autoptic series have shown that a vast majority of PE are not diagnosed ante-mortem [7-9]. More recently, the issue of potential overdiagnosis has also been raised, following the development and widespread use of sensitive, albeit less specific, diagnostic tools [10].

It has been recognized for a long time that symptoms and signs of PE have low sensitivities and specificities $[11,12]$. This observation must not be taken to imply that accurate history and physical examination are not important, but rather that they must be used in a rigorous approach, in combination with laboratory findings and imaging techniques, to maximize the accuracy of the diagnosis.

\section{The suspicion of PE}

Based on the obvious principle that a disease cannot be recognized if it is not considered among the diagnostic possibilities, raising the suspicion of $\mathrm{PE}$ is the first step in the work-up of this disease [13]. At this stage, ideally one would want to achieve a very high sensitivity in order to include all cases that might benefit from a prompt treatment; only later would a more stringent analysis be needed to avoid an excessive number of more costly (both from and economical and a biological point of view) tests. Thus, any sign, symptom, or result of a diagnostic test that has been shown to be associated with PE should be used for the sole purpose of raising the suspicion and enroll the patient in a more rigorous work-up.

Combining data from numerous studies undertaken to recognize clinical features suggestive of PE, including our own [14], a recent meta-analysis has provided a list of risk factors, signs and symp- 
Table 1. - Predisposing factors, signs and symptoms associated with PE (modified from [15])

\begin{tabular}{lcc}
\hline Feature & Likelihood ratio & 95\% confidence interval \\
\hline Predisposing factors & & \\
\hline Thrombophlebitis & 2.20 & $0.44-3.29$ \\
Current deep vein thrombosis & 2.05 & $1.12-3.73$ \\
Active cancer & 1.74 & $1.17-2.59$ \\
Surgery & 1.63 & $1.23-1.12$ \\
Past history of venous thromboembolism & 1.47 & $1.11-1.96$ \\
Immobilization & 1.41 & $1.20-1.60$ \\
Estrogen therapy & 1.26 & \\
\hline Symptoms & & \\
\hline Syncope & & $1.84-1.81$ \\
Sudden dyspnoea & 2.38 & $1.07-3.13$ \\
Haemoptysis & 1.83 & $1.23-1.12$ \\
Leg pain & 1.62 & $0.94-2.74$ \\
Dyspnoea & 1.60 & $1.14-1.78$ \\
Chest pain & 1.42 & $0.86-1.33$ \\
\hline Signs & 1.07 & \\
\hline Shock & & \\
Leg swelling & & $1.84-8.96$ \\
Tachypnea & & $1.59-2.79$ \\
Cyanosis & 4.07 & $1.15-1.57$ \\
Tachycardia & 2.11 & $0.69-2.63$ \\
& 1.34 & $0.94-1.88$ \\
\hline
\end{tabular}

toms associated with PE [15]. Table 1 lists all the features with likelihood ratios (LR) greater than 1 , i.e. more frequent in EP patients than in patients without the disease. It is important to note than some of the features have LR barely above 1 (e.g. chest pain, LR 1.07; 95\% CI 0.86-1.33) and that the single symptom with the highest LR is syncope with a LR of 2.38 (95\% CI 1.54-3.69), thus confirming the relatively poor performance of clinical data in the diagnostic process. Once again, these features are nevertheless of utmost importance in order to raise the suspicion of PE and submit the patient to further diagnostic tests. Since respiratory alkalosis with hypoxemia is a common, albeit non specific, feature in PE $[16,17]$ an otherwise unexplained, sudden hypocarbic hypoxemia can also be used to raise the suspicion of the disease.

Finally, an increase in the circulating levels of D-dimer, a fibrin degradation product whose role in the diagnostic process of PE will be discussed in greater detail later, has been proposed as a legitimate reason to suspect $\mathrm{PE}$ in a young individual with no apparent reasons for increased activation of the coagulation cascade, such as recent surgery, injury, trauma, or pregnancy [18]; however, rigorous studies on this topic are not available at this time.

\section{The assessment of clinical probability}

Once a disease has been suspected, and therefore included in the differential diagnosis, the typical further step would be to submit the patient to a definitive diagnostic test, with an accuracy deemed sufficient to confirm or rule out the diagnosis. With the possible exception of pulmonary arteriography, that would not be available for the majority of patients due to its biological and economic costs [19], such test does not exist for PE. Rather, it has been repeatedly demonstrated that the best approach is to combine an accurate assessment of the probability of the disease estimated on the basis of clinical features (the so called clinical or pre test probability) with the results of further laboratory or imaging tests [20,21].

Numerous studies have addressed the issue of what is the best way to calculate the clinical probability. While clinical experience and judgment are irreplaceable, in this as is most medical fields, predefined scores have been proposed in order to improve the accuracy of the assessment, particularly by less experienced physicians. Currently, three such scores have been validated in a sufficient number of patients to be of clear clinical utility: the Canadian, or Wells, score [22] (table 2), the revised Geneva score [23] (based on a previous, more complex one [24]) (table 3), and the PISAPED score [20] (table 4). While showing important differences, all three approaches divide patients into low, intermediate and high probability of disease. Further simplifications of these rules have been attempted, but at present are still awaiting prospective evaluation $[25,26]$. All these rules have potential pitfalls, and none is currently considered clearly better than the others. This situation has brought a certain lack of confidence in the 
Table 2. - Clinical prediction rules for PE: the Wells score [22]

\begin{tabular}{lc}
\hline Variable & Points \\
\hline Previous deep vein thrombosis or PE & 1.5 \\
Recent surgery or immobilization & 1.5 \\
Cancer & 1 \\
Haemoptysis & 1 \\
Heart rate $>100$ beats/min & 1.5 \\
Clinical signs of deep vein thro mbosis & 3 \\
Alternative diagnosis less likely than PE & 3 \\
\hline Clinical Probability & Total \\
\hline Low & $0-1$ \\
Intermediate & $2-6$ \\
High & $>7$ \\
\hline
\end{tabular}

Table 3. - Clinical prediction rules for PE: the revised Geneva score [23]

\begin{tabular}{lc}
\hline Variable & Points \\
\hline Age > 65 years & 1 \\
Previous deep vein thrombosis or PE & 3 \\
Surgery or fracture within a month & 2 \\
Active malignancy & 2 \\
Unilateral lower limb pain & 3 \\
Haemoptysis & 2 \\
Heart rate 75-94 beats/min & 3 \\
Heart rate > 95 beats/min & 5 \\
Pain on lower limb deep vein at palpation & 4 \\
and unilateral edema & Total \\
\hline Clinical Probability & $0-3$ \\
\hline Low & $4-10$ \\
Intermediate & $>11$ \\
\hline High
\end{tabular}

application of this rigorous approach, likely limiting its use in clinical practice. Furthermore, the continuous attempts to modify, improve and simplify these scores make it difficult to complete and compare the prospective validation studies that are much needed for a widespread diffusion of this approach.

\section{D-dimer}

Dimerization of $\mathrm{D}$ regions originating from two fibrinogen molecules requires conversion of fibrinogen into fibrin and subsequent polymerization of fibrin; for D-dimers to be released into the blood stream, the activation of the fibrinolytic system is then required. Thus, the presence of circulating D-dimers reflects the activation of the coagulation and fibrinolytic cascades. Accordingly, circulating D-dimer levels have been tested as potential markers of thromboembolic disorders [27]. Because numerous conditions can activate the coagulation and fibrinolytic systems, it is not surprising that D-dimer levels have shown a high sensitivity and a low specificity in the diagnostic work-up of suspected PE. Using an ELISA assay in a series of outpatients with suspected PE, Perrier and coworkers have shown that a cut-off of $500 \mathrm{ng} / \mathrm{mL}$ discriminates PE from non PE patients with an overall sensitivity of $99.5 \%$ and a specificity of $41 \%$ [28]. Based on these data, a role for low levels of $\mathrm{D}$-dimers in ruling out the diagnosis of PE has been proposed and extensively validated. The current positioning of Ddimer testing will be discussed in greater detail in the final paragraph; however, it is important to notice that the performance of the test varies greatly depending on the laboratory method used. Less expensive and/or faster methods, suitable in the setting of an emergency room for example, are available but show a lower overall accuracy compared to the standard ELISA assay [29]. It is therefore of critical importance that physicians that prescribe D-dimer testing in the work-up of suspected PE are aware of the actual assay used in the laboratory.

Table 4. - Clinical prediction rules for PE: the PISA-PED score [20]

High probability:

presence of at least one of three symptoms (sudden onset dyspnoea, chest pain, or fainting) not explained otherwise and associated with: (1) any two of the following abnormalities: electrocardiographic signs of RV overload, radiographic signs of oligemia, amputation of hilar artery, or pulmonary consolidations compatible with infarction; (2) any one of the above three radiographic abnormalities.

Intermediate probability:

presence of at least one of the above symptom s, not explained otherwise, but not associated with the above electrocardiographic and radiographic abnormalities, or associated with electrocardiographic signs of right ventricular overload only.

Low probability:

absence of the above three symptoms, or identification of an alternative diagnosis that may account for their presence (e.g., exacerbation of COPD, pneumonia, lung edema, myocardial infarction, pneumothorax, and others). 


\section{Imaging: Lung scintigraphy}

Ventilation/Perfusion (V/Q) lung scan has been the mainstay of the diagnosis of PE for decades [30]. In principle, the sensitivity of the perfusion scan is very high, since any areas where blood flow is arrested by an embolus would appear as perfusion defects. Accordingly, a normal perfusion scan excludes PE with a negative predictive value close to $100 \%$ [31]. On the other hand, because other conditions besides PE, including tumors, pneumonic consolidations, areas of disrupted pulmonary circulation due to emphysema, etc., potentially cause perfusion defects, the specificity of the perfusion scan is low. The addition of the ventilation scan was proposed in an attempt to increase specificity, on the assumption that areas with simultaneous reduction of perfusion and ventilation (matching defects) would reflect the presence of primarily parenchymal conditions, while only mismatching defects would be characteristic of PE [30]. While this approach has rapidly gained widespread diffusion, particularly in the US, its limits have also become increasingly clear. Sensitivity and specificity, based on the simplified reading categories proposed by the so called PIOPED II trial ("PE present", "PE absent", and "nondiagnostic") are 78\% and 98\%, respectively, after excluding nondiagnostic readings; however, the latter are very frequent, representing $27 \%$ of total [32]. Also, the very pathophysiological rationale of the V/Q scan has been recently questioned on the basis of the existence of physiological adaptation mechanisms whereby ventilation is diverted from underperfused areas to normally perfused areas to minimize ventilation/perfusion inequality and therefore improve gas exchange [33]. Furthermore, the reabsorption of surfactant in unperfused regions leads to the generation of areas of atelectasis that are also excluded from ventilation [33]. Such phenomena would therefore greatly limit the utility of the distinction between matching and mismatching defects. Based on the above considerations, many centers, including our own [14], have favored the use of perfusion scans without ventilation; chest $\mathrm{X}$-rays are used in order to recognize areas of parenchymal involvement responsible for perfusion defects. This approach has been best formalized in the PISA-PED trial [34]. Besides its alleged theoretical advantages, avoiding ventilation scans also reduces radiation burden, patients' discomfort, and costs. In a recent retrospective analysis, data and images from the PIOPED II trial were read using the PISA-PED criteria [35]. Perfusion scans combined with chest X-ray provided diagnostic accuracy similar to V/Q scans and computed tomography (CT) angiography (see below) with lower costs and radiation burdens. Of particular interest, no perfusion scans were nondiagnostic, compared to $6 \% \mathrm{CT}$ angiographies and $21 \%$ V/Q scans. These results prompted the Authors to conclude that "ventilation scans are superfluous in most patients" [35]. It is likely that future studies on different diagnostic algorithms for PE will incorporate perfusion scans in combination with chest X-ray rather than V/Q scans.

\section{Imaging: Computed tomography}

Spiral CT with i.v. injection of an iodinated contrast material (CT angiography, CTA) [36] has emerged as a very useful tool in the diagnostic work-up of patients with suspected PE and has in fact rapidly become the primary imaging test at many institutions. Early concern about the low sensitivity of single slice CTA [37] has been overcome by the use of multi-detector scanners. Based on data from the PIOPED II trial, obtained with 4, 8 , or 16 row multi-detector scanners, the sensitivity and specificity of CTA were $83 \%$ and $96 \%$, respectively; $6 \%$ studies were inconclusive due to poor image quality [38]. As mentioned before, these figures are similar to those reported for perfusion lung scan combined with chest X-ray, according to the PISA-PED criteria, in the same data set (sensitivity $80 \%$; specificity $97 \%$ ), although no lung scans were considered inconclusive [35]. Of note, $22 \%$ of patients had contraindications to CTA either due to elevated creatinine or to allergy to iodinated contrast material. The sensitivity of the test could be increased to $90 \%$ by adding the venous phase imaging of the proximal leg veins (CT venography, CTV), based on the notion that $\mathrm{PE}$ and deep vein thrombosis represent manifestations of one pathologic process; addition of CTV, however, also increased the proportion of inconclusive studies to $11 \%$ [38], as well as the radiation burden to the patient (see below). It must be stressed that this study clearly showed that the accuracy of CTA, either alone on in combination with CTV, depends heavily on the assessment of clinical probability. While the positive predictive value of a positive CTA in the presence of high clinical probability (as assessed by the Canadian rule) was $96 \%$, it dropped to $58 \%$ (57\% when CTV was taken into account) in patients with low clinical probability. Similarly, the negative predictive value of a negative CTA went from $96 \%$ (97\% in combination with CTV) in the presence of low clinical probability to $60 \%$ (82\% in combination with CTV) in patients with high clinical probability. These figures clearly demonstrate that CTA/CTV are of little value without an appropriate estimate of the clinical probability.

As mentioned before, CTA has rapidly gained widespread use, and available data on its diagnostic accuracy certainly support its use. Furthermore, when negative for PE, CTA has the advantage of offering information on other conditions potentially responsible for the patient's symptoms, although prospective studies aimed at assessing the actual impact of this added value on the patients' outcome are not available. Finally, it is likely that the accuracy of CTA will increase with the development of more sophisticated scanners. One concern that has recently emerged, however, is the radiation exposure associated with CTA/CTV. The calculated effective whole-body dose for a CTA 
varies from 1.6 to $8.3 \mathrm{mSv}$, while CTV reportedly causes an effective whole body dose of $5.7 \mathrm{mSv}$. Thus, CTA/CTV would cause a radiation exposure 7 to 15 -fold higher than a perfusion scan combined with standard two-view chest $\mathrm{X}$ ray $(0.9 \mathrm{mSv})$. The absorbed dose to the female breast with CTA has been calculated as 10-50 mGy, compared to $0.28 \mathrm{mGy}$ with a perfusion lung scan; for comparison, the absorbed dose to the breast with standard two-view mammography is $3 \mathrm{mGy}$ [39].

\section{Diagnostic algorithms}

Based on the sensitivity, specificity, positive and negative predictive values, costs, potential risks and local availability of the various diagnostic tools, different diagnostic algorithms have been proposed.

The diagnostic work-up begins with the suspicion of PE, raised on the basis of the presence of some of the signs, symptoms and risk factors associated with the disease. Once PE has been considered in the differential diagnosis, the clinical probability should be formally assessed, using one of the available rules. If the clinical probability, as assessed by either the Canadian or the revised Geneva score, is low, a D-dimer test should be ordered. It has been clearly demonstrated that in the presence of a "negative" D-dimer test (i.e. a value below the defined threshold for the specific assay method used) it is safe to withhold anticoagulation [40-42]. The situation is more complicated when the clinical probability is intermediate, since only "high sensitivity", quantitative D-dimer assay methods are considered sensitive enough to rule out the diagnosis of PE in intermediate probability patients [43]. As mentioned before, physicians should be aware of the actual assay method available in the laboratory. In patients with high clinical probability, the D-dimer test should not be ordered, because no result, irrespective of the sensitivity of the method, is deemed sufficient to safely rule out the diagnosis [43]. No studies have investigated the role of D-dimer in combination with the clinical probability as assessed by the PISA-PED score.

Patients with a "positive" D-dimer test, irrespective of the clinical probability, or patients with high probability (and intermediate probability when a sensitive D-dimer test is not available) should undergo further tests [44]. CTA (with or without CTV) is commonly considered the imaging test of choice. The performance of CTA in terms of sensitivity and specificity has been recently shown to be similar to that of the combination of perfusion lung scan and chest X-ray [35]. Of note, when first introduced, CTA was compared to V/Q scan $[45,46]$ a test whose diagnostic power is hampered by the high percentage of non conclusive results. This, and the wide availability of CTA in most emergency departments, probably contribute to explain the success of this technique, despite its inherently higher radiation burden and potential risks. Perfusion lung scan represents the first choice imaging technique in patients with contraindications to CTA, such as high creatinine levels and allergy to iodinated contrast material. The hypothesis that lung scan is also preferable in younger women, due the lower absorbed dose to the breast, awaits formal testing. In practice, a negative multi-detector CTA is considered an adequate criterion for excluding $\mathrm{PE}$ if the clinical probability is low or intermediate. Patients with high clinical probability and a negative CTA should undergo perfusion lung scan based on the insufficient negative predictive value of CTA in this situation [38]. Conversely, a positive CTA is adequate proof of $\mathrm{PE}$ in patients with high or intermediate clinical probability. In patients with low clinical probability, the best approach depends on the level of the clot visualized by the CTA. If the clot is in the lobar or main pulmonary artery, anticoagulation is warranted [44]; in patients with low clinical probability and a segmental clot, a perfusion lung scan should be obtained. One important practical implication of this last recommendation is that if segmental or subsegmental clots are incidentally recognized at a CT ordered for other reasons in a patient with no clinical suspicion of $\mathrm{PE}$, further tests must be obtained before a final diagnosis is reached.

Where isotope lung scanning is readily available, a perfusion scan coupled with chest X-ray can be used as the first-line imaging technique. If the clinical and isotope scan results are concordant, a definitive diagnosis is possible; otherwise, CTA must be obtained [47].

The PISA-PED investigators have proposed an alternative diagnostic algorithm that does not rely on the D-dimer test, even in patients with low clinical probability [20]. According to this approach, a perfusion lung scan is obtained in all patients. In case of high or intermediate clinical probability, as assessed by the PISA-PED own score, and a positive lung scan, the diagnosis of $\mathrm{PE}$ is confirmed; in the presence of low clinical probability and a negative scan the diagnosis is ruled out; in case of discordant clinical/scintigraphic outcome, further testing is required. In the original PISA-PED series, less than $20 \%$ patients actually required further testing. This method appears particularly useful for inpatients, in which the D-dimer test is usually abnormal and therefore of little diagnostic value.

In conclusion, no diagnostic algorithm is clearly superior to the others, and the choice reflects personal experience and local availability of diagnostic tests; however, it is important to use the rigorous, standardized approaches described, in order to minimize the risk of misdiagnosis.

\section{References}

1. Oger E. Incidence of venous thromboembolism: a community-based study in Western France. EPI-GETBP Study Group. Groupe d'Etude de la Thrombose de Bretagne Occidentale. Thromb Haemost 2000; 83: 657-660.

2. Heit JA, Melton L Jr, Lohse CM, et al. Incidence of venous thromboembolism in hospitalized patients vs com- 
munity residents. Mayo Clin Proc 2001; 76: 1102-1110.

3. Giuntini C, Di Ricco G, Marini C, Melillo E, Palla A Pulmonary embolism: epidemiology. Chest 1995; 107: 3S-9S.

4. Kearon C, Kahn SR, Agnelli G, Goldhaber S, Raskob GE, Comerota AJ. Antithrombotic therapy for venous thromboembolic disease: American College of Chest Physicians Evidence-Based Clinical Practic e Guidelines (8th Edition). Chest 2008; 133: 454S-545S.

5. Barritt DW, Jordan SC. Anticoagulant drugs in the treatment of pulmonary embolism. A controlled trial. Lancet 1960; 1: 1309-1312.

6. Schulman S, Beyth RJ, Kearon C, Levine MN. Hemorrhagic complications of anticoagulant and thrombolytic treatment: American College of Chest Physicians Evidence-Based Clinical Practice Guidelines (8th Edition). Chest 2008; 133: 257S-298S.

7. Goldhaber SZ, Hennekens CH, Evans DA, Newton EC, Godleski JJ. Factors associate d with correct antemortem diagnosis of major pulmonary embolism. Am J Med 1982; 73: 822-826.

8. Rubinstein I, Murray D, Hoffstein V. Fatal pulmonary emboli in hospitalized patients. An autopsy study. Arch Intern Med 1988; 148: 1425-1426.

9. Sandler DA, Mar tin JF. Autopsy proven pulmonary embolism in hospital patients: are we detecting enough deep vein thrombosis? J R Soc Med 1989; 82: 203-205.

10. Bounameaux H, Perrier A. Diagnosis of pulmonary embolism: in transition. Curr Opin Hematol 2006; 13: 344-350.

11. Hildner FJ, Ormond RS. Accuracy of the clinical diagnosis of pulmonary embolism. JAMA 1967; 202: 567 570 .

12. Moser KM. Venous thromboembolism. Am Rev Respir Dis 1990; 141: 235-249.

13. Palla A, Petruzzelli S, Donnamaria V, Giuntini C. The role of suspicion in the diagnosis of pulmonary embolism. Chest 1995; 107: 21S-24S.

14. Celi A, Palla A, Petruzzelli S, et al. Prospective study of a standardized questionnaire to improve clinical estimate of pulmonary embolism. Chest 1989; 95: 332-337.

15. West J, Good acre S, Sampson F. The value of clinical features in the diagnosis of acute pulmonary embolism: systematic review and meta-analysis. QJM 2007; 100 : 763-769.

16. Green RM. Pulmonary embolism in younger adults. Chest 1992; 101: 1507-1511.

17. Prediletto R, Miniati M, Tonelli L, et al. Diagnostic value of gas exchange tests in patients with clinical suspicion of pulmonary embolism. Crit Care 1999; 3: 111116.

18. Lippi G, Franchini M, Targher G, Favaloro EJ. Help me, Doctor! My D-dimer is raised. Ann Med 2008; 112.

19. Stein PD, Athanasoulis C, Alavi A, et al. Complications and validity of pulmonary angiography in acute pulmonary embolism. Circulation 1992; 85: 462-468.

20. Miniati M, Prediletto R, Formichi B, et al. Accuracy of clinical assessment in the diagnosis of pulmonary embolism. Am J Respir Crit Care Med 1999; 159: 864871.

21. van Belle A, Buller HR, Huisman MV, et al. Effectiveness of managing suspected pulmonary embolism using an algorithm combining clinical probability, D-dimer testing, and computed tomography. JAMA 2006; 295 : 172-179.

22. Wells PS, Anderson DR, Rodger M, et al. Derivation of a simple clinical model to categorize patients probability of pulmonary embolism: increasing the models utility with the SimpliRED D-dimer. Thromb Haemost 2000; 83: 416-420.

23. Le Gal G, Righini M, Roy PM, et al. Prediction of pul- monary embolism in the emergency department: the revised Geneva score. Ann Intern Med 2006; 144: 165171.

24. Wicki J, Perneger TV, Junod AF, Bounameaux H, Perrier A. Assessing clinical prob ability of pulmonary embolism in the emergency ward: a simple score. Arch Intern Med 2001; 161: 92-97.

25. Gibson NS, Sohne M, Kruip MJ, et al. Further validation and simplification of the Wells clinical decision rule in pulmonary embolism. Thromb Haemost 2 008; 99: 229-234.

26. Miniati M, Bottai M, Monti S, Salvadori M, Serasini L, Passera M. Simple and accurate prediction of the clinical probability of pulmonary embolism. Am J Respir Crit Care Med 2008; 178: 290-294.

27. Prisco D, Grifoni E. The role of D-dimer testing in patients with suspected venous thromboembolism. Semin Thromb Hemost 2009; 35: 50-59.

28. Perrier A, Desmarais S, Goehring C, et al. D-dimer testing for suspected pulmonary embolism in outpatients. Am J Respir Crit Care Med 1997; 156: 492-496.

29. Stein PD, Hull RD, Patel KC, et al. D-dimer for the exclusion of acute venous thrombosis and pulmonary embolism: a systematic review. Ann Intern Med 2004; 140: 589-602.

30. McNeil BJ, Holman BL, Adelstein SJ. The scintigraphic definition of pulmonary embolism. JAMA 1974; 227: 753-756.

31. van Beek EJ, Kuyer PM, Schenk BE, Brandjes DP, ten Cate JW, Buller HR. A normal perfusion lung scan in patients with clinically suspected pulmonary embolism. Frequency and clinical validity. Chest 1995; 108: 170173.

32. Sostman HD, Stein PD, Gottschalk A, Matta F, Hull R, Goodman L. Acute pulmonary embolism: sensitivity and specificity of ventilation-perfusion scintigraphy in PIOPED II study. Radiology 2008; 246: 941-946.

33. Giuntini C. Ventilation/perfusion scan and dead space in pulmonary embolism: are they useful for the diagnosis? Q J Nucl Med 2001; 45: 281-286.

34. Miniati M, Pistolesi M, Marini C, et al. Value of perfusion lung scan in the diagnosis of pulmonary embolism: results of the Prospective Investigative Study of Acute Pulmonary Embolism Diagnosis (PISA-PED). Am J Respir Crit Care Med 1996; 154: 1387-1393.

35. Sostman HD, Miniati M, Gottschalk A, Matta F, Stein PD, Pistolesi M. Sensitivity and specificity of perfusion scintigraphy combined with chest radio graphy for acute pulmonary embolism in PIOPED II. $J$ Nucl Med 2008; 49: 1741-1748.

36. Layish DT, Tapson VF. New imaging techniques for the diagnosis of pulmonary embolism. Curr Opin Pulm Med 1997; 3: 280-285.

37. Rathbun SW, Raskob GE, Whitsett TL. Sensitivity and specificity of helical computed tomography in the diagnosis of pulmonary embolism: a systematic review. Ann Intern Med 2000; 132: 227-232.

38. Stein PD, Fowler SE, Goodman LR, et al. Multidetector computed tomography for acute pulmonary embolism. N Engl J Med 2006; 354: 2317-2327.

39. Stein PD, Woodard PK, Weg JG, et al. Diagnostic pathways in acute pulmonary embolism: recommendations of the PIOPED II Investigators. Radiology 2007; 242: $15-21$.

40. Wells PS, Anderson DR, Rodger M, et al. Excluding pulmonary embolism at the bedside without diagnostic imaging: management of patients with suspected pulmonary embolism presenting to the emergency department by using a simple clinical model and D-dimer. Ann Intern Med 2001; 135: 98-107.

41. Kelly J, Hunt BJ. A clinical probability assessment and D-dimer measurement should be the initial step in the 
investigation of suspected venous thromboembolism. Chest 2003; 124: 1116-1119.

42. Righini M, Aujesky D, Roy PM, et al. Clinical usefulness of D-dimer depending on c linical probability and cutoff value in outpatients with suspected pulmonary embolism. Arch Intern Med 2004; 164: 2483-2487.

43. Roy PM, Colombet I, Durieux P, Chatellier G, Sors H, Meyer G. Systematic review and meta-analysis of strategies for the diagnosis of suspected pulmonary embolism. BMJ 2005; 331: 259.

44. Torbicki A, Perrier A, Konstantinides S, et al. Guidelines on the diagnosis and management of acute pulmonary embolism: the Task Force for the Diagnosis and Management of Acute Pulmonary Embolism of the
European Society of Cardiology (ESC). Eur Heart $J$ 2008; 29: 2276-2315.

45. Blachere H, Latrabe V, Montaudon M, et al. Pulmonary embolism revealed on helical CT angiography: comparison with ventilation-perfusion radionuclide lung scanning. Am J Roentgenol 2000; 174: 1041-1047.

46. Coche E, Verschuren F, Keyeux A, et al. Diagnosis of acute pulmonary embolism in outpatients: comparison of thin-collimation multi-detector row spiral CT and planar ventilation-perfusion scintigraphy. Radiology 2003; 229: 7 57-765.

47. British Thoracic Society guidelines for the management of suspected acute pulmonary embolism. Thorax 2003; 58: $470-483$.

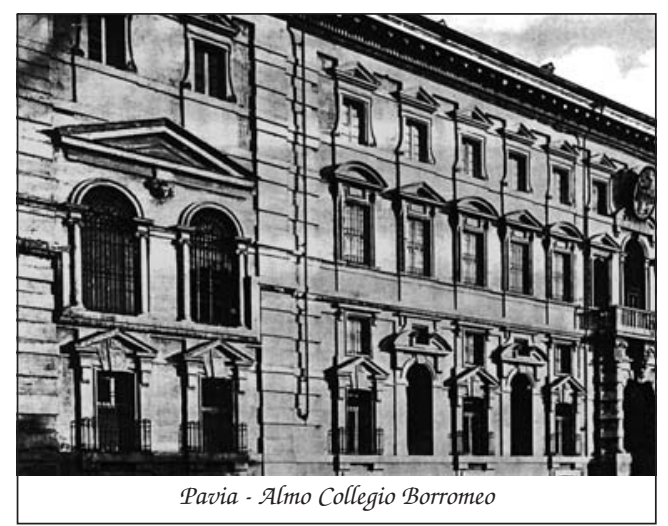

